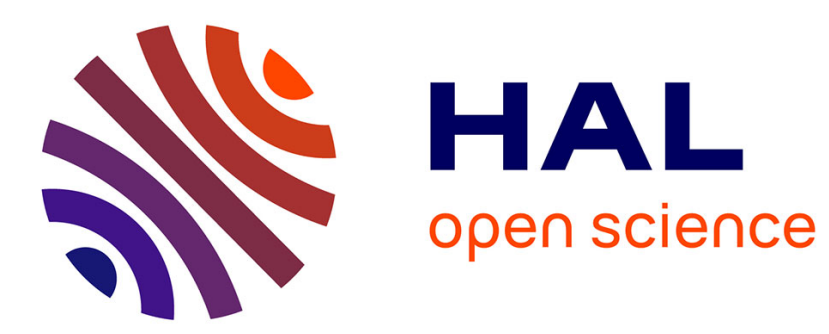

\title{
Motion field of curves: applications
}

Théodore Papadopoulo, Olivier Faugeras

\section{To cite this version:}

Théodore Papadopoulo, Olivier Faugeras. Motion field of curves: applications. [Research Report] RR-2168, INRIA. 1994. inria-00074504

\section{HAL Id: inria-00074504 https://hal.inria.fr/inria-00074504}

Submitted on 24 May 2006

HAL is a multi-disciplinary open access archive for the deposit and dissemination of scientific research documents, whether they are published or not. The documents may come from teaching and research institutions in France or abroad, or from public or private research centers.
L'archive ouverte pluridisciplinaire HAL, est destinée au dépôt et à la diffusion de documents scientifiques de niveau recherche, publiés ou non, émanant des établissements d'enseignement et de recherche français ou étrangers, des laboratoires publics ou privés. 


\section{Motion field of curves : applications}

Théo Papadopoulo, Olivier Faugeras

\section{$\mathbf{N}^{\circ} \mathbf{2 1 6 8}$}

Janvier 1994

PROGRAMME 4

Robotique,

image

et vision

\section{apport \\ derecherche}





\title{
Motion field of curves : applications
}

\author{
Théo Papadopoulo*, Olivier Faugeras** \\ Programme 4 - Robotique, image et vision \\ Projet Robotvis
}

Rapport de recherche $\mathrm{n}^{\circ} 2168$ - Janvier $1994-37$ pages

\begin{abstract}
This report discusses the well known problem of structure from motion for the special case of rigid curves. It is already known that it is theoretically possible to recover the motion and then the structure of a moving $3 \mathrm{D}$ rigid curve observed Cin a monocular image sequence as soon as some set of derivatives that are defined on the so-called spatio-temporal surface can be computed. This is true under the most general camera model of perspective projection. We give here a new simplification of the previous results. However, this theory is still quite difficult to implement. In order to show that this implementation is indeed feasible, we proceeded towards two main directions. First, we have implemented the special case of planar rigid curves which turns out to be much more tractable and quite informative. As a step towards the general case, we show that the necessary derivatives defined on the spatio-temporal surface can indeed be computed from the images : one of the main practical difficulty for the implementation of the theory is thus solved. In order to demonstrate the accuracy of the results and the effectiveness of the implementations, we experiment on both synthetic and real image sequences.
\end{abstract}

Key-words: motion analysis, structure from motion, rigid curves, spatio-temporal surfaces, computer vision

(Résumé : tsvp)

The research described in this report has been supported by DRET contract $\mathrm{N}^{\circ} 911$ 349/A00 and by ESPRIT BRA project Insight II

*papadop@sophia.inria.fr

**faugeras@sophia.inria.fr

Unité de recherche INRIA Sophia-Antipolis

2004 route des Lucioles, BP 93, 06902 SOPHIA-ANTIPOLIS Cedex (France)

Téléphone : (33) 93657777 - Télécopie : (33) 93657765 


\section{Applications du champ de mouvement des courbes}

Résumé : Ce rapport traite du problème de l'obtention de la structure d'une scène à partir d'une séquence d'images dans le cas particulier des courbes rigides. Il est maintenant connu qu'il est possible théoriquement de retrouver un tel mouvement, et par suite sa structure, en observant cette courbe rigide à l'aide d'une caméra si l'on sait calculer un certain jeu de paramètres défini sur la surface spatio-temporelle engendrée par cette courbe. Ce résultat est vrai dans le cadre générique de la vision par ordinateur où le modèle sténopé a été adopté pour la caméra. Nous donnons ici une nouvelle simplification des résultats précédents. Cependant, cette théorie reste encore difficile à implémenter. Afin de démontrer la faisabilité d'une telle implémentation, nous avons avancé dans deux directions complémentaires: tout d'abord nous avons traité jusqu'au bout le cas particulier des courbes rigides planes qui se trouve être plus aisé à implémenter et fournit de précieux enseignements. Par ailleurs, nous montrons qu'il est possible de calculer à partir des images les dérivées spatio-temporelles nécessaires à l'implémentation du cas général: ainsi, une des principales difficultés pratiques de l'implémentation de la théorie est résolue. Afin de montrer la précision des résultats obtenus ainsi que l'efficacité des implémentations, nous avons testé celles-ci à l'aide de séquences d'images aussi bien synthétiques que réelles.

Mots-clé : analyse du mouvement, structure à partir du mouvement, courbes rigides, surface spatio-temporelle, vision par ordinateur 


\section{Introduction}

Recovering three-dimensionnal information about an observed scene from images is one of the main goals of computer vision. Many methods have been designed to give an answer to this question. The basic underlying idea leading to a vast majority of these methods is to combine information coming from many different view points.

The more successful methods are those based on stereo [MK85, II86, PH86, AL87, RF91] : two or more (usually three) views of the scene taken at the same time instant are combined to obtain the $3 \mathrm{D}$ coordinates of the observed points. However, for this method to work, it is necessary to know the internal parameters of the cameras and their relative positions in any $3 \mathrm{D}$ frame. These parameters can be obtained either offline by a process called camera calibration [Tsa86, FT86] which supposes that some known pattern is observed or dynamically by a process called auto-calibration that uses the Euclidean structure of the observed $3 \mathrm{D}$ world to compute the cameras parameters from point correspondences [FLM92, HGC92].

Alternatively, another way to obtain multiple viewpoints is to use motion [FLT87, WKPS87, FDN89, SA90]. In these approaches only one camera is used, thus only the internal parameters of the camera are needed which simplifies the calibration procedure. From the measurement of the motion of image primitives (flow fields), the $3 \mathrm{D}$ motion parameters and the relative depth can be computed. However, there are many ways to compute flow fields : most of these approaches are based on optical flow which is computed directly from image intensities [LHP80, HS81, Nag83, Hil84, Koe86, D'H86, Bou89, Gon89]. Another way to proceed is to use the motion fields in the image which are defined as the projection in the image retina of the 3D motion field of some geometric object (usually points or lines). [FP92] studies the relationship between optical flow and motion field for general 3D curves and shows that the assumptions made in the computation of the optical flow are a bit difficult to defend. Moreover, it shows that for a rigid 3D curve it is theoretically possible to recover the 3D structure and motion from a monocular sequence of images. The data needed in order to achieve these tasks are derivatives defined on the so-called spatio-temporal surface. In order to implement these ideas, we focussed on three main directions :

$\operatorname{RR} n^{\circ} 2168$ 
- Simplify the existing equations.

- Find methods to compute the needed derivatives.

- Implement some simpler cases, namely the case of planar 3D curves.

After the introduction of notations, we examine briefly why the equations for the general case can be simplified and how to relate the 3D motion of planar curves to the first order derivatives in space and time on the spatio-temporal surface. Then, we will describe how it is possible to verify the computed motion when using real images and how to use this same method to disambiguate curve matches with a stereo rig for which only the internal parameters are known. In the final section, we will discuss our implementation for the planar curve case and results on both synthetic and real images. As well, results of the derivative computation needed for the general monocular case will be presented. 


\section{Notations and Basic Results}

The goal of this section is to introduce some notations relative to the problem of recovering the $3 \mathrm{D}$ motion from $2 \mathrm{D}$ motion fields. We will also recall some basic results that are described in details in [FP92] and explain without proof why one of the basic results of this paper can be simplified.

\subsection{The Camera Model}

We assume that the camera obeys the pinhole model as shown in Fig. 1. The model composes of a retina plane $\mathcal{R}$ and of a focal center point $O$. The retina $\mathcal{R}$ is parallel to the plane $(O, X, Y)$ and is at distance $f$ of $O$ where $f$ is the focal distance. By applying an adequate scaling on the coordinates $(X, Y, Z)$, it is always possible to suppose that $f=1$. The unit focal length (normalized to 1 ) is therefore assumed in the the discussion to follow.

The frame $(O, X, Y, Z)$ being naturally attached to the camera model, all equations involving $3 \mathrm{D}$ parameters will be written in this frame.

Given a $3 \mathrm{D}$ point $\mathbf{M}=(X, Y, Z)$ and its $2 \mathrm{D}$ perspective projection $\mathbf{m}=$ $(x, y, 1)$ on the $\mathcal{R}$ plane, their relationship is characterized $\mathrm{b}$ the following equation :

$$
\mathbf{M}=Z \mathbf{m}
$$

This equation is fundamental in that all the constraints we will present here are direct consequences of it. The concept of temporal variance can be incorporated with the introduction of a time factor $\tau$.

\subsection{Definitions}

We now assume that we observe in a sequence of images a family $\left(c_{\tau}\right)$ of curves, where $\tau$ denotes time, which we assume to be the perspective projection in the retina of a $3 \mathrm{D}$ curve $(C)$ that moves in space. If we consider the threedimensional space $(x, y, \tau)$, this family of curves sweeps in that space a surface $(\Sigma)$ defined as the set of points $\left(\left(c_{\tau}\right), \tau\right)$. Figure 2 illustrates an example of 


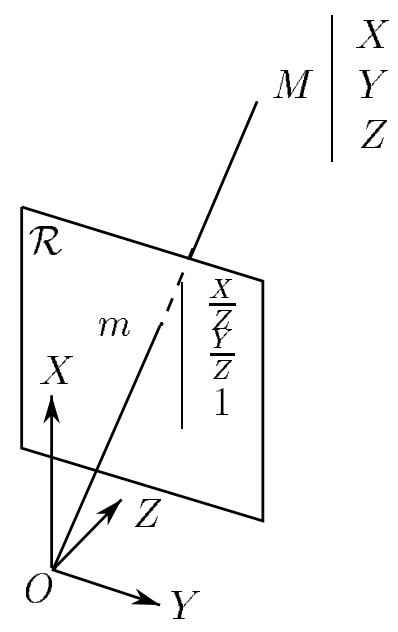

Figure 1: The pinhole model of a camera

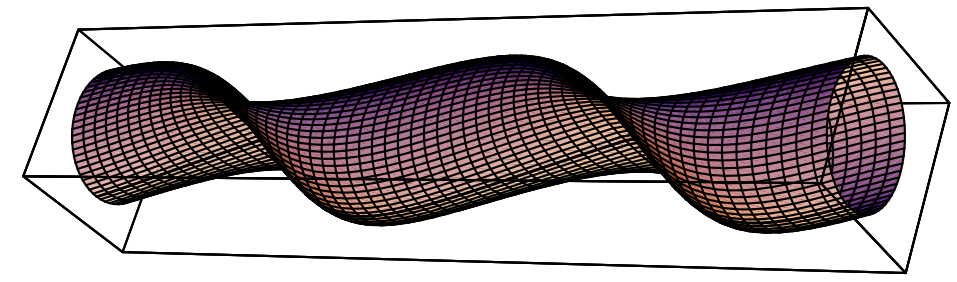

Figure 2: The spatio-temporal surface generated by a circle rotating in front of the camera 
one such spatio-temporal surface generated by a circle rotating around one of its diameters in front of the camera.

At a given time instant $\tau$, let $s$ be the arclength of $\left(c_{\tau}\right)$ and $S$ the arclength of $(C)$. We further suppose that $S$ is not a function of time (i.e. the motion is isometric). Now, for a point $\mathbf{m}$ on $\left(c_{\tau}\right)$, it is possible to define two different motion fieds :

- The apparent motion field $\mathbf{v}_{m}^{a}$ (a for apparent) of $\mathbf{m}(s, \tau)$ is the partial derivative with respect to time when $s$ is kept constant, $\frac{\partial \mathbf{m}}{\partial \tau}=\mathbf{m}_{\tau}$.

- The real motion field $\mathbf{v}_{m}^{r}$ ( $r$ for real) is the partial derivative of $\mathbf{m}(s, \tau)$ with respect to time when $S$ is kept constant, or its total time derivative $\dot{\mathbf{m}}$. This field is the projection of the $3 \mathrm{D}$ velocity field in the retina.

Moreover, introducing the Frenet frame $(\mathbf{t}, \mathbf{n})$, where $\mathbf{n}$ is the unit normal vector to $\left(c_{\tau}\right)$ at $\mathbf{m}$, we have

$$
\begin{array}{r}
\mathbf{v}_{m}^{a}=\alpha \mathbf{t}+\beta \mathbf{n} \\
\mathbf{v}_{m}^{r}=w \mathbf{t}+\beta \mathbf{n}
\end{array}
$$

where $\alpha$ is the tangential apparent motion field, $w$ is the tangential real motion field and $\beta$ is the normal motion field. Note that $\beta$ has a nice geometric interpretation as shown in Fig. 3. We consider the normal $\mathbf{n}$ at point $m$ of curve $\left(c_{\tau}\right)$. At time $\tau+d \tau$, the curve $c_{\tau+d \tau}$ is intersected by the line defined by $m$ and $\mathbf{n}$ at a point $p$ represented by $\mathbf{m}+\beta \mathbf{n} d \tau$.

With these notations, under the weak assumption of isometric motion, we reach the following conclusions from the study of the spatio-temporal surface :

1. The normal motion field $\beta$ can be recovered from the normal to the spatio-temporal surface,

2. the tangential apparent motion field can be recovered from the normal motion field,

3. the tangential real motion field cannot be recovered from the spatiotemporal surface.

$\operatorname{RR} n^{\circ} 2168$ 


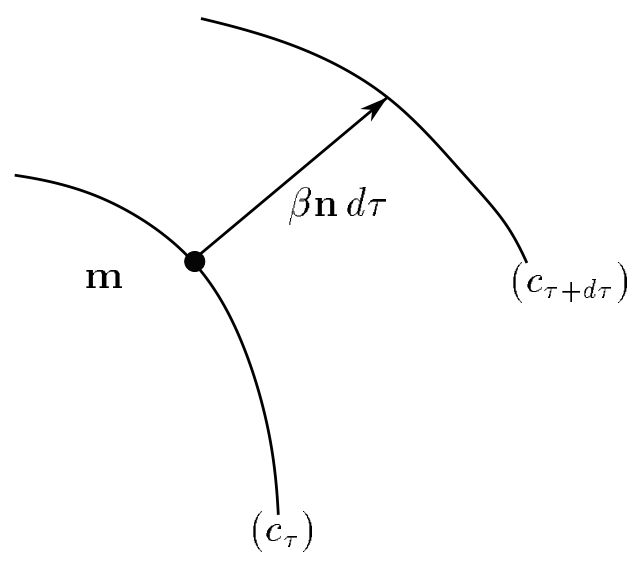

Figure 3: A geometric interpretation of $\beta$

Therefore, the full real motion field is not computable from the observation of the image of a moving curve under the isometric assumption. This can be considered as a new statement of the so-called aperture problem. In order to solve it we must add more constraints, for example that the $3 \mathrm{D}$ motion is rigid.

\subsection{The Case of a Rigid 3D Curve}

Assuming now that the curve $(C)$ is moving rigidly. Let $(\boldsymbol{\Omega}, \mathbf{V})$ be its kinematic screw at the optical center $O$ of the camera. We assume also that the camera has been normalized by calibration to unit focal length. Finally, recall that the velocity $\dot{\mathbf{M}}$ of any point $\mathbf{M}$ attached to the rigid body is given by

$$
\mathbf{V}_{M}=\dot{\mathbf{M}}=\mathbf{V}+\Omega \times \mathbf{M}
$$

where the vector $\mathbf{V}_{M}$ is the three-dimensional velocity of point $M$.

Taking the total derivative of (1) with respect to time, we get :

$$
V_{M_{Z}} \mathbf{m}+Z(w \mathbf{t}+\beta \mathbf{n})=\mathbf{V}_{M}
$$

Projecting this vector equation onto $\mathbf{t}$ and $\mathbf{n}$ yields two scalar equations : 


$$
\begin{gathered}
Z(w+\boldsymbol{\Omega} \cdot \mathbf{b})=-\mathbf{U}_{\mathbf{n}} \cdot \mathbf{V} \\
Z(\beta-\mathbf{\Omega} \cdot \mathbf{a})=\mathbf{U}_{\mathbf{t}} \cdot \mathbf{V}
\end{gathered}
$$

where $\mathbf{U}_{\mathbf{t}}$ denotes $\mathbf{m} \times \mathbf{t}$ and $\mathbf{U}_{\mathbf{n}}$ denotes $\mathbf{m} \times \mathbf{n}$ and $\mathbf{a}$ and $\mathbf{b}$ are given by :

$$
\begin{aligned}
\mathbf{a} & =\mathbf{m} \times \mathbf{U}_{\mathbf{t}} \\
\mathbf{b} & =\mathbf{m} \times \mathbf{U}_{\mathbf{n}}
\end{aligned}
$$

These equations are fundamental (especially (4)) in the sense that they express the relationship between the unknown $3 \mathrm{D}$ motion of a point and the real motion field of its image.

Let's now recall some previous result that is given in [FP92].

Théorème 1 At each point of an observed curve $\left(c_{\tau}\right)$ evolving during time, it is possible to write two polynomial equations in the coordinates $\boldsymbol{\Omega}, \mathbf{V}, \dot{\boldsymbol{\Omega}}$ and $\dot{\mathbf{V}}$ (The kinematic screw attached to the $3 D$ curve and its first time derivative). The coefficients of these equations are polynomials in the quantities

$$
\begin{array}{ccccc}
\beta & \frac{\partial \beta}{\partial s} & \frac{\partial^{2} \beta}{\partial s^{2}} & \partial_{\mathbf{n}_{\beta} \beta} \beta & \partial_{\mathbf{n}_{\beta}} \frac{\partial \beta}{\partial s} \\
\kappa & \frac{\partial \kappa}{\partial s} & \partial_{\mathbf{n}_{\beta}} \kappa &
\end{array}
$$

that can be measured from the spatio-temporal surface $(\Sigma)$.

These equations lead to a way to compute the motion and the structure of the $3 \mathrm{D}$ curve. It can be shown that they are, in fact, a direct consequence of (4) and that one of these equations is redundant because it can be expressed as a linear combination of the other equation and its first time derivative. Thus the previous result can be restated as :

Théorème 2 At each point of an observed curve $\left(c_{\tau}\right)$ evolving during time, it is possible to write one polynomial equation in the coordinates $\mathbf{\Omega}, \mathbf{V}, \dot{\boldsymbol{\Omega}}$ and $\dot{\mathbf{V}}$ (The kinematic screw attached to the $3 D$ curve and its first time derivative). The coefficients of this equation are polynomials in the quantities

$$
\beta \frac{\partial \beta}{\partial s} \partial_{\mathbf{n}_{\beta}} \beta
$$

that can be measured from the spatio-temporal surface $(\Sigma)$.

$\operatorname{RR~}{ }^{\circ} 2168$ 
The nice thing with this new theorem is that we get rid of all third order derivatives with only first and second order derivatives being left. Therefore we are only interested in the above-mentionned derivatives and we will show later that it is possible to compute them quite precisely. 


\section{The Motion of 3D Planar Rigid Curves}

We study here a special case of the motion of rigid curves sketched in the previous section : the case of a 3D rigid curve that is planar. By making this hypothesis, it is possible to write an equation similar to those obtained in the general case but :

- in which $\dot{\boldsymbol{\Omega}}$ and $\dot{\mathbf{V}}$ are no longer involved, thus leading to a system of equations with less unknowns.

- of total degree 2 (instead of 4$)$.

- in which only the first order derivatives of $\Sigma$ will appear.

The first two characteristics show that the systems we obtain is much simpler (less unknowns with lower degrees), thus the number of possible solutions is smaller. The second characteristic means that not only the equation are simpler but also they are more stable with respect to the measurement noise. We thus may hope that the solutions of the system are also more stable. These two reasons have appeared sufficently attractive to motivate us to implement this case before going to the general case.

Basically, all these simplifications are possible because of the introduction of new unknowns that correspond to the normal $\mathbf{N}=[a, b, c]^{T}$ to the curve plane.

\subsection{The Equation in the Planar Case}

Let $\mathbf{M}=[X, Y, Z]^{T}$ be a point on the $3 \mathrm{D}$ planar curve. This point belongs to the plane of the curve. If we suppose that the image of the curve is not degenarated into a segment, then the optical center of the camera is not on the curve plane. The point $\mathbf{M}$ thus verifies the following equation :

$$
\mathbf{N} \cdot \mathbf{M}+1=a X+b Y+c Z+1=0
$$

Combining (5) with the perspective equation (1), we obtain :

$$
Z \mathbf{N} \cdot \mathbf{m}+1=0
$$

$\operatorname{RR} n^{\circ} 2168$ 
or :

$$
Z=\frac{-1}{\mathbf{N} \cdot \mathbf{m}}
$$

Equation (6) is fundamental because it connects the plane structure of the curve $Z$ to the measures in the image $x, y$. Now remember (4) :

$$
Z(\beta-\mathbf{\Omega} \cdot \mathbf{a})=\mathbf{U}_{\mathbf{t}} \cdot \mathbf{V}
$$

Replacing $Z$ by its value given by (6) in (4), we obtain :

$$
-\frac{\beta-\Omega \cdot \mathbf{a}}{\mathbf{N} \cdot \mathbf{m}}=\mathbf{U}_{\mathbf{t}} \cdot \mathbf{V}
$$

Equation (6) is essential : it allows one to replace the quantity $Z$ that varies along the observed curve by 3 quantities $a, b, c$ that are constant along this same curve. We thus obtain the following theorem :

Théorème 3 At each point of $\left(c_{\tau}\right)$ considered as the projection of a $3 D$ planar curve, it is possible to write a polynomial equation in the unknowns $\boldsymbol{\Omega}, \mathbf{V}, \mathbf{N}$.

$$
\beta-\mathbf{\Omega} \cdot \mathbf{a}+\left(\mathbf{U}_{\mathbf{t}} \cdot \mathbf{V}\right)(\mathbf{N} \cdot \mathbf{m})=0
$$

This equation is not homogeneous in $\mathbf{V}$ but we can see that if $(\mathbf{V}, \mathbf{N})$ is a solution then $\left(\lambda \mathbf{V}, \frac{1}{\lambda} \mathbf{N}\right)$ is also a solution for every $\lambda \neq 0$. This property shows that, as in the general case, only the direction of $\mathbf{V}$ can be recovered. The equation is of degree 2 in $(\mathbf{V}, \mathbf{N})$, of degree 1 in $\mathbf{\Omega}$ and of total degree 2.

In the following we will call this equation the planar equation.

Expressing this equation at 8 points, we obtain a system in $(\boldsymbol{\Omega}, \mathbf{V}, \mathbf{N})$ of degree 2 with 9 unknowns. A direct application of Bezout's theorem shows that such a system has $2^{8}=256$ complex solutions.

It is then possible to reformulate the conjecture we have made in the previous section for this particular case :

Conjecture 1 The kinematic screw $(\boldsymbol{\Omega}, \mathbf{V})$ and the normal $\mathbf{N}$ to the plane of a $3 D$ rigid planar curve can, in general, be estimated from the observation of the spatio-temporal surface generated by its image on the retina by solving a 
system of polynomial equations. The quantity $Z$ can be estimated at each point up to a scale factor by using (6).

Of course, as in the general case, this conjecture is wrong in some special cases such as straight lines but also, as we will see later, conics. See [Ber89] for other examples of ambiguity. Practically, for non ambiguous curves, this conjecture has always been proved to be true.

\subsection{Ambiguousness of the Solutions}

We are interested here in describing the structure of the solutions. The question is : is it possible generically to find a relation between two solutions (here this means that the result we look for does not depend on the actual values of the estimated parameters). One way to do this is to search for a transformation on $(\boldsymbol{\Omega}, \mathbf{V}, \mathbf{N})$ that leaves the equation unchanged.

Let us thus look at the coefficients of $(8)$ in variables $x, y, \mathbf{t}, \beta$. We obtain :

$$
\begin{array}{rc}
\text { coefficient in } \beta: & 1 \\
\text { coefficient in } \mathbf{t}_{x}: & \boldsymbol{\Omega}_{x}+c \mathbf{V}_{y} \\
\text { coefficient in } \mathbf{t}_{y}: & \boldsymbol{\Omega}_{y}-c \mathbf{V}_{x} \\
\text { coefficient in } x \mathbf{t}_{x}: & a \mathbf{V}_{y}-\boldsymbol{\Omega}_{z} \\
\text { coefficient in } y \mathbf{t}_{y}: & -\boldsymbol{\Omega}_{z}-b \mathbf{V}_{x} \\
\text { coefficient in } x \mathbf{t}_{y}: & c \mathbf{V}_{z}-a \mathbf{V}_{x} \\
\text { coefficient in } y \mathbf{t}_{x}: & b \mathbf{V}_{y}-c \mathbf{V}_{z} \\
\text { coefficient in } x\left(x \mathbf{t}_{y}-y \mathbf{t}_{x}\right): & \boldsymbol{\Omega}_{y}+a \mathbf{V}_{z} \\
\text { coefficient in } y\left(x \mathbf{t}_{y}-y \mathbf{t}_{x}\right): & b \mathbf{V}_{z}-\boldsymbol{\Omega}_{x}
\end{array}
$$

These different terms represent the way the information relative to $(\boldsymbol{\Omega}, \mathbf{V}, \mathbf{N})$ is coded in the (8) and this is independent of the point at which the equation is written. In some way, every quantity that cannot be computed from these terms or that remains ambiguous will exhibit the same behaviour when computed from the (8). We will use this property to prove that there is a companion solution to each solution of the system.

$\operatorname{RR} n^{\circ} 2168$ 
It is possible to eliminate the components of $\boldsymbol{\Omega}$ between $(9),(10),(11),(12)$ and $(13),(14)$. We thus obtain the following expressions:

$$
\begin{aligned}
& b \mathbf{V}_{z}+c \mathbf{V}_{y} \\
& c \mathbf{V}_{x}+a \mathbf{V}_{z} \\
& a \mathbf{V}_{y}+b \mathbf{V}_{x} \\
& c \mathbf{V}_{z}-a \mathbf{V}_{x} \\
& c \mathbf{V}_{z}-b \mathbf{V}_{y}
\end{aligned}
$$

Note that it is possible to swap $\mathbf{V}$ and $\mathbf{N}$ in these expressions without changing their values. Let us look if it is possible to find a value of $\boldsymbol{\Omega}$ that verifies the equations. By equating the new values with the old ones, we obtain :

$$
\begin{aligned}
& \boldsymbol{\Omega}_{x}^{\prime}+b \mathbf{V}_{z}=\boldsymbol{\Omega}_{x}+c \mathbf{V}_{y} \\
& \boldsymbol{\Omega}_{y}^{\prime}+c \mathbf{V}_{x}=\boldsymbol{\Omega}_{y}+a \mathbf{V}_{z} \\
& \boldsymbol{\Omega}_{z}^{\prime}+a \mathbf{V}_{y}=\boldsymbol{\Omega}_{z}+b \mathbf{V}_{x}
\end{aligned}
$$

Solving this system in $\boldsymbol{\Omega}^{\prime}$, we see that we must take $\boldsymbol{\Omega}^{\prime}=\boldsymbol{\Omega}+\mathbf{V} \wedge \mathbf{N}$.

We thus obtain the following theorem :

Théorème 4 If $(\boldsymbol{\Omega}, \mathbf{V}, \mathbf{N})$ is a solution of the system obtained for a planar curve then $(\boldsymbol{\Omega}+\mathbf{V} \wedge \mathbf{N}, \mathbf{N}, \mathbf{V})$ is also a solution of this same system.

This theorem is the specialization to planar curves of a well-known theorem on planar points [LH84, May92]. This is not surprising since a planar curve is nothing more than a set of planar points. What is more surprising however is that it can be shown that there is no new ambiguity introduced by the fact we only use normal flow information. Expressions (9-16) can also be used to find a better bound to the number of solutions : since (8) is linear in these expressions, by expressing this equation at 8 points it is possible generically to find unique values for each of the (9-16). Then solving for $(\boldsymbol{\Omega}, \mathbf{V}, \mathbf{N})$ it is possible to show that there is at least one and at most 3 solutions (counting 
only once the two related solutions) which is a much better bound than that computed from Bezout's theorem.

To conclude this section, let us briefly present some results concerning a special case of $3 \mathrm{D}$ planar curves : conics and algebraic planar curves.

\subsection{The Case of Conics}

When the observed curve is, at each time instant, a conic, on can prove that the $3 \mathrm{D}$ curve associated to it is also a conic. It is thus possible to apply our theory for planar curves to this more specific case. Moreover there is a standard mathematical technique, called elimination, that can be used here to write directly all the equations that can be written along the conic. This will give a finite set of algebraic equations instead of an (theoretically) infinite set of equations at each point of the conic. Elimination is a technique of suppressing a variable between two polynomials that vanish simultaneously. Here one polynomial is (8) and the other is the equation of the conic. Both equations depend on the retinal coordinates $x$ and $y$. Thus eliminating one of these variables (for example $y$ ) between these two polynomials, we obtain a new polynomial in one variable $(x)$ that must vanish everywhere. Thus all the coefficients of this polynomial (considered as a polynomial in $x$ ) must be zero. These coefficients are new polynomials in $\boldsymbol{\Omega}, \mathbf{V}$ and $\mathbf{N}$. These new polynomials relate globally the observed conic to the motion of the $3 \mathrm{D}$ conic. Doing this one obtains a system of 5 equations with 8 unknowns. This proves definitively that the observation of a conic without making any other hypothesis is insufficient for recovering the $3 \mathrm{D}$ motion. Actually it is possible to give an arbitrary value to $\mathbf{V}$ (or equivalently $\mathbf{N}$ ) to get a linear system with 5 equations and 6 unknowns $(\boldsymbol{\Omega}, \mathbf{N})$ (respectively $(\boldsymbol{\Omega}, \mathbf{V})$ ) which generically gives always a unique solution. Thus the observation of a conic gives rise to a triple infinity of different $3 \mathrm{D}$ motions (and thus also 3D structures). Moreover, there is no way to obtain some new constraint (by, for example, deriving once again the obtained equations) without assuming something on either the motion or on the conic.

Note that the scheme that was sketched here is valid for any given alge-

braic curve. Then for a generic algebraic curve of degree $n$, we obtain $\frac{n(n+3)}{2}$ equations. It is interesting to note that this number is exactly the number of 
$2 \mathrm{D}$ points necessary to define the curve uniquely. This result shows us that for any $3 \mathrm{D}$ generic rigid planar curve whose projection is an algebraic curve of degree higher than 3 , there will be a finite set of solutions. 


\section{How to Use $(\Omega, V)$}

The main goal of this section is to show what can be done once $(\boldsymbol{\Omega}, \mathbf{V})$ has been computed : one obvious thing to do is to recover the $3 \mathrm{D}$ structure of the curve but it is also possible to compute quantities that are independent of the frame in which $(\boldsymbol{\Omega}, \mathbf{V})$ has been computed. This is most interesting to help verifying quantitatively the quality of the results when dealing with real images as well as for combining information coming from many different cameras.

\subsection{Reconstruction of the Curve}

From previous formulas, once the motion is computed, it is possible to reconstruct the $3 \mathrm{D}$ curve up to a scale factor by two different means :

- Using (5) which relates $Z$ to the plane parameters $\mathbf{N}$. Here we are using explicitely the planar hypothesis.

- Using the general (4) that is true for all $3 \mathrm{D}$ rigid curve. It relates $Z$ to the kinematic screw $(\boldsymbol{\Omega}, \mathbf{V})$. Since we do not use the planar hypothesis, the reconstructions computed this way are more unstable than the previous ones.

\subsection{Motion Invariants}

The goal of this section is twofold :

- Show how it is possible to verify quantitatively the quality of the results. Usually when experimenting with real images, it is possible to know precisely the $3 \mathrm{D}$ motion in some frame that cannot be related easily to the camera frame. Thus the easiest way to achieve this goal is to compare quantities depending on $(\boldsymbol{\Omega}, \mathbf{V})$ that are invariant to this Euclidean change of frame.

- Expanding on this idea, if a scene is observed by a stereo rig for which only the internal parameters are known, it is possible to disambiguate curve matches as soon as the candidate curves are undergoing motions

$\operatorname{RR} n^{\circ} 2168$ 
different enough. This has to be compared with the method for calibrated stereo rigs that has been sketched in [FP92].

To achieve this goal, let's recall two basic properties of the kinematic screw.

- The component $\boldsymbol{\Omega}$ is independant of the point at which the kinematic screw is expressed whereas $\mathbf{V}$ depends on it.

- The quantity $\boldsymbol{\Omega} \cdot \mathbf{V}$ is also independent of the point at which the kinematic screw is expressed even though $\mathbf{V}$ is not. It expresses the values of the translationnal velocity along the instantaneous rotation axe.

But, since we don't know the frame in which the 3D motion is measured and since $\mathbf{V}$ is known only up to a scale factor, we cannot directly rely on these quantities. However, the norm of $\boldsymbol{\Omega}$ as well as the angle between $\boldsymbol{\Omega}$ and $\mathbf{V}$ are invariant to any change of frame and can thus be used as the searched quantitative measure for veryfing the quality of the results using real images.

So far, we have not used $\mathbf{N}$ but it is possible for the planar case to derive some other measures namely $\boldsymbol{\Omega} \cdot \mathbf{N}$ and $\mathbf{V} \cdot \mathbf{N}$.

Using a stereo rig with known internal parameters, it is also possible to use these quantities to disambiguate stereo matches for curves with different underlying motions. Suppose you have computed the kinematic screws $\left(\boldsymbol{\Omega}_{\mathbf{1}}, \mathbf{V}_{\mathbf{1}}\right)$ and $\left(\boldsymbol{\Omega}_{\mathbf{2}}, \mathbf{V}_{\mathbf{2}}\right)$ respectively for a curve $\left(C_{1}\right)$ in camera 1 and for a curve $\left(C_{2}\right)$

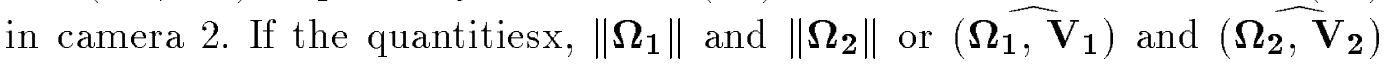
disagree then the curve match is wrong. If they agree, however, it is possible to match the reconstructions $R_{1}$ and $R_{2}$ to verify the matching hypothesis and to improve the quality of the recovered $3 \mathrm{D}$ structure since the two structures $R_{1}$ and $R_{2}$ are related by a similitude in that case. 


\section{Implementation}

We describe here the implementation of the theory described in the previous section and show the results we have obtained on both synthetic and real images.

In all these experiments the first stage is to adapt the camera model we have hypothesized to derive the valid equations for the real camera. This is explained in the next section.

\subsection{Normalized Coordinates and Image Coordinates}

In the previous sections, we have assumed that a Euclidean structure is defined on the retinal plane $\mathcal{R}$. This structure is inherited from the $3 \mathrm{D}$ Euclidean structure. This hypothesis allows us to write scalar and cross products that are necessary to obtain the equations. In fact, if one considers the normal flow, it immediately leads to the notion of angle, which is closely tied to scalar product.

However, when working with images, people are used to, for convinience, to work in a frame in which the coordinates of a pixel correspond to the indices of that pixel in the image considered as a two dimensionnal array of pixels. These coordinates are called image coordinates. Working with these coordinates has two consequences:

- First, the usual scalar product does not define in this frame an Euclidean structure compatible with that of the observed 3D space.

- Secondly, the origin of these frames are usually in the top left corner of the image whereas in our theoretical study that origin is at the orthogonal projection (in $3 \mathrm{D}$ space) of the optical center onto the retina $\mathcal{R}$.

The consequence of these is that, before doing anything else, it is necessary to map the Euclidean structure of the $3 \mathrm{D}$ space onto the retina $\mathcal{R}$. This is equivalent of being able to define a special scalar product on the retina that is comptatible with that of $3 \mathrm{D}$ space. One way of doing this is to transform

$\operatorname{RR} n^{\circ} 2168$ 
the image coordinates to obtain some normalized coordinates with which the standard scalar product behaves the right way.

Relations between image and normalized coordinates have been extensively studied under the framework of camera calibration. We will utilize the commonly used camera calibration model as defined in [Tos87] :

$$
\begin{aligned}
& x=h_{1} u+h_{2} v+h_{3} \\
& y=h_{4} v+h_{5}
\end{aligned}
$$

where $x, y$ denote the normalized coordinates of a point and $u, v$ denote image coordinates of this same point. $\left(h_{1}, h_{2}, h_{3}, h_{4}, h_{5}\right)$ are the internal parameters of the camera. The change of coordinates defined by (17) and (18) must be applied before any motion computation.

Note that we have assumed here that the transformation between normalized and image coordinates is time independent. We have assumed as well that the period of time between two consecutive images is constant and known precisely.

\subsection{Solving the Polynomial System}

We have seen that, in order to find the 3D motion of the planar curve, we need to solve an over-constrained polynomial system. It is crucial for both finding real solutions and dealing with noise in the measures, to have a numerical stable method that allows us to work with over-constrained polynomial systems (that is more equations than unknowns). Generally speaking this kind of systems has no solutions but we know that our systems are in that very special case where (in the absence of noise) there is at least one solution (the actual motion of the 3D curve).

Moreover, experience that are not described here have convinced us that, in practise, the number of solutions is quite small and that there is a good chance to find the good one considering any reasonable initial estimate. Since we are unable (neither theoretically nor practically) to count the number of real solutions of the system, we are not actually able to find all of them. Instead we 
will focus on a method that allows us to compute a solution given any random starting point. Some other experiments not described here allow us to believe that tracking this solution through time bring always us back to the searched for solution or to its companion as described in Theorem 4.

One obvious choice to solve this problem is to use least-squares. However, since our equations are non-linear this introduces many fictious solutions (depending on the degrees of the equations in the unknowns and on the number of these unknowns). Therefore we decided to modify the multi-dimensional Newton method to adapt it to our requirements. In this method, the hypothesis of having exactly the same number of equations as the number of unknowns is used only to inverse the jacobian of the system (thus we need a square jacobian matrix, which means that there must be as many unknowns as equations). Since this inversion is only needed to solve a linear system, we may apply leastsquare methods directly on this linear system that as exactly the same number of equations and the same number of unknowns as the initial system. Since this system is linear we know that least squares will not introduce spurious solutions and this seems much better than directly applying least squares on the original system.

We tested this method on a system of equations chosen among the equations of a linear family of circles and it gave good results even when we added noise onto the equations. It showed good properties of convergence and stability.

\subsection{Motion of Planar Curves}

Many tests have shown that it is difficult to estimate with a good accurate spatio-temporal parameters. Temporal derivatives are especially difficult to obtain : this phenomenon seems to come from sampling problems in time. Whereas spatial sampling of an image may be known and constant (it is fixed by the physical parameters of the camera), time sampling of the spatio-temporal surface $\Sigma$ around a point $P$ depends on the speed of that point. In other words, around a point $P$ that has a small normal flow, $\Sigma$ is better sampled than around a point where the normal flow is important. The importance of this fact on temporal derivatives is illustrated in Fig. $3:$ as the curves $\left(c_{\tau}\right)$ and

$\left(c_{\tau+d \tau}\right)$ move further apart, the sampling of surface $\Sigma$ in the direction of $\mathbf{n}_{\beta}$

$\operatorname{RR~}{ }^{\circ} 2168$ 
deteriorates. Temporal derivatives can be obtained very easily by considering the curve drawn on $\Sigma$ that lies in the plane defined by the point $\mathbf{m}$ at which we want to compute the spatio-temporal parameters, and being spanned by the vectors $\mathbf{n}$ the normal vector to the observed curve at $\mathbf{m}$ and by $\tau$ the unit vector on the time axis. Therefore, the accuracy of the temporal derivatives depends upon how well this curve is sampled. This is the reason why we have implemented first the case of planar curves since it involves only first order derivatives whereas second order derivatives are needed to solve the general case.

In the remaining of this paper, we will suppose that the points of the curve that we are observing have been linked together into pixel chains. Moreover, we will use two image sequences (of about 30 to 40 images each). The first one (see Fig. 4) is a synthetic sequence of a planar 3D quartic on which intensity images we added up to $20 \%$ of noise on intensities. The second one (see Fig. 5) is a real sequence. In all these images the $3 \mathrm{D}$ curve rotates around a vertical axis and translates in the same direction.

Here is the general scheme of the implementation :

- Building the spatio-temporal surface.

- Estimating the spatio-temporal parameters at every point of the curve at one time instant.

- Normalising the parameters and finally solving of the polynomial system.

Each of these stages will be described in the next paragraphs.

\subsection{Building the Spatio-Temporal Surface}

In order to speed up computation times, we must gather the points of the spatio-temporal surface in a data structure that will allow us to compute easily the neighbours of a given point on the surface. To do this, we will take the ouput of the linker and map its result in a doubly linked list of points. In order to recover on some errors of the edge detector we link points that are not contiguous with some threshold. During this work a curvilinear abscissa 

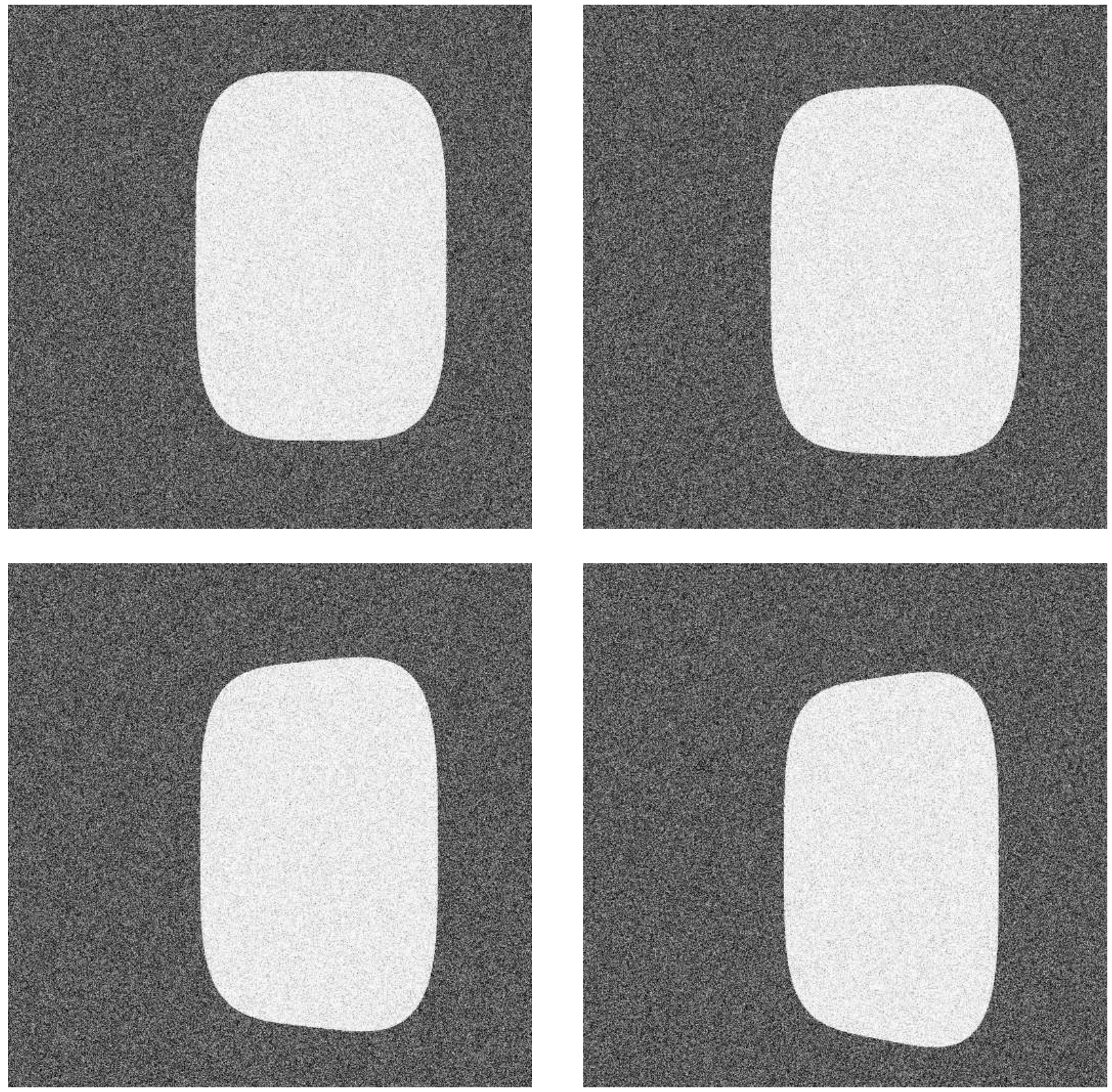

Figure 4: From right to left and top to bottom : the images of the synthetic sequence at instants $0,10,20$ and 30 . A Gaussian noise of signal/noise ratio of $20 \%$ has been added on intensities.

$\operatorname{RR} n^{\circ} 2168$ 

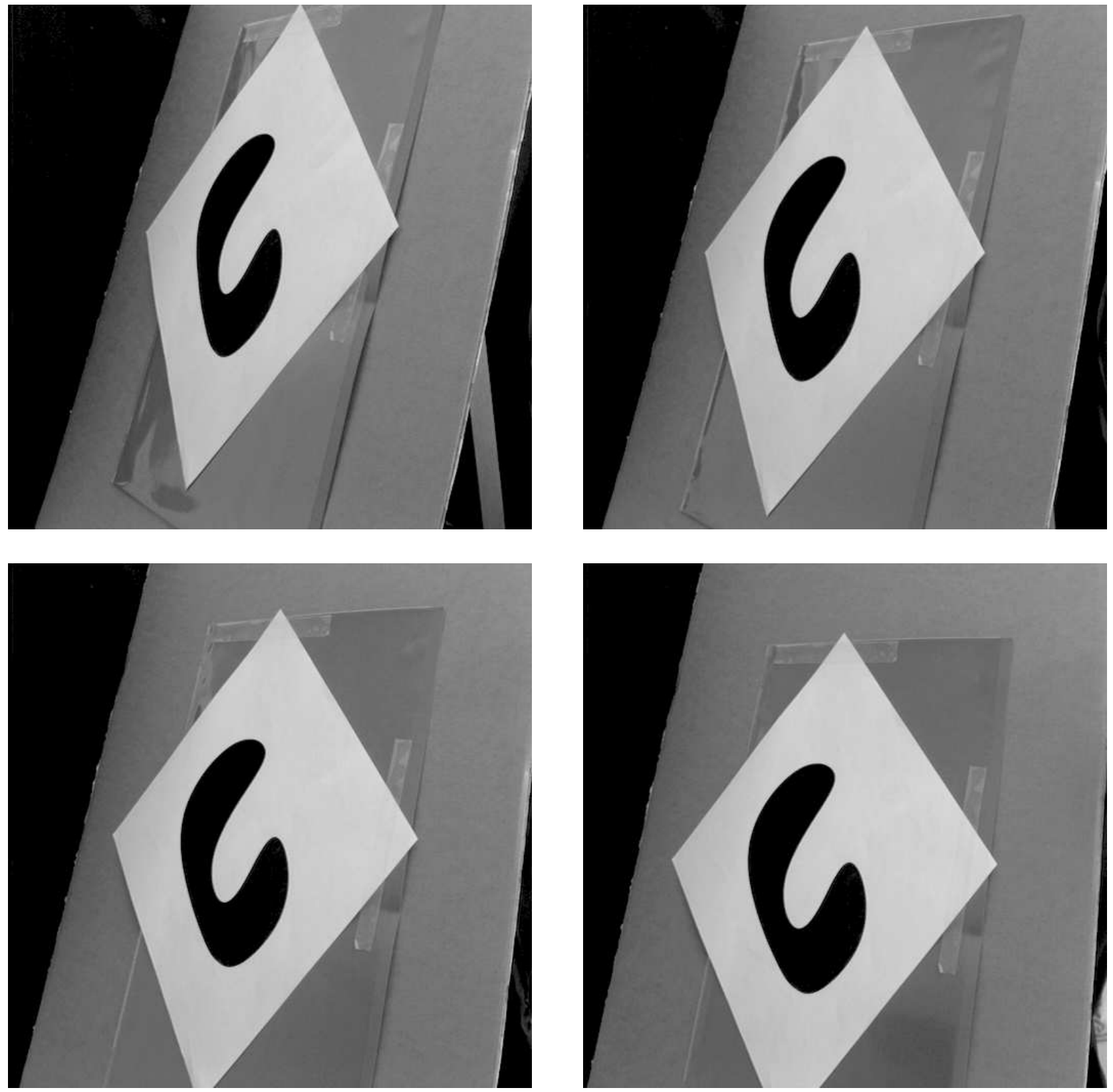

Figure 5: From right to left and top to bottom : the images of the second real sequence at instants $1,10,20$ and 30 . 
is computed for each point by choosing an arbitrary origin and by addind the elementary distances between points along the edge. A second phase consists in linking the data structure obtained for the curve $\left(c_{\tau}\right)$ (observed at time $\tau$ ) to that of the curve $\left(c_{\tau+d \tau}\right)$ (observed at time $\left.\tau+d \tau\right)$. To do this at each point $\mathbf{m}_{\tau}$ of the curve $\left(c_{\tau}\right)$, we associate one of the points of $\left(c_{\tau+d \tau}\right)$ that is at minimal distance from $\mathbf{m}_{\tau}$ (because of discretization there might be several such points but they are all in a same neighborhood. We are chosing one of these arbitrarily). The algorithm attempts to use the continuity of the curves to avoid walking through to many points of either $\left(c_{\tau}\right)$ or $\left(c_{\tau+d \tau}\right)$. This time linking is done simultaneously in the two directions : from $\left(c_{\tau}\right)$ to $\left(c_{\tau+d \tau}\right)$ and from $\left(c_{\tau+d \tau}\right)$ to $\left(c_{\tau}\right)$. This give us a two-way link in time.

\subsection{Estimating Spatio-Temporal Parameters}

The estimation of the spatio-temporal parameters is just sketched here. Because of the discrepancy between space and time sampling rates we will compute independently spatial and temporal parameters. First we compute the local orientation at each point : to do so, we construct the two signals $x(s)$ and $y(s)$ and fit locally models to them. Deriving these models gives the local derivatives $x^{\prime}(s)$ and $y^{\prime}(s)$ that describe the local tangent to the curve which is closely related to the orientation represented here by the angle $\theta(s)$ between the normal and the horizontal. Then, we eventually smooth this result.

The left part of Fig. 6 shows the angle estimates along the curve. The maximal error between the theoric curve and the measures is 0.011 radians.

We then compute the value of the $\beta$ parameter at each point. To reach this goal, we use the method described at the begining of this section. At each point $\mathbf{m}$, which orientation is given by $\mathbf{n}$, we build the curve that is defined as the intersection of the spatio-temporal surface $\Sigma$ with the plane $\Pi$ defined by $\mathbf{m}, \mathbf{n}$ and $\tau$ the time axis. This curve can be represented in the plane $(\tau$, where $d$ is the distance in the direction of $\mathbf{n}$ between a point of plane $\Pi$ and $\mathbf{m}$ (this distance is also the distance of this point to the tangent to $\left(c_{\tau}\right)$ at $\mathbf{m}$ if we represent $\left(c_{\tau}\right)$ and $\left(c_{\tau+d \tau}\right)$ in the same plane). We thus obtain a curve that is approximated by a truncated Chebyshev polynomial (to reduce the effects of noise). The derivative of this polynomial at time $\tau$ is then computed and is nothing other than $\beta$. Again, we smooth the curve $\beta(s)$ in order to

$\operatorname{RR} n^{\circ} 2168$ 

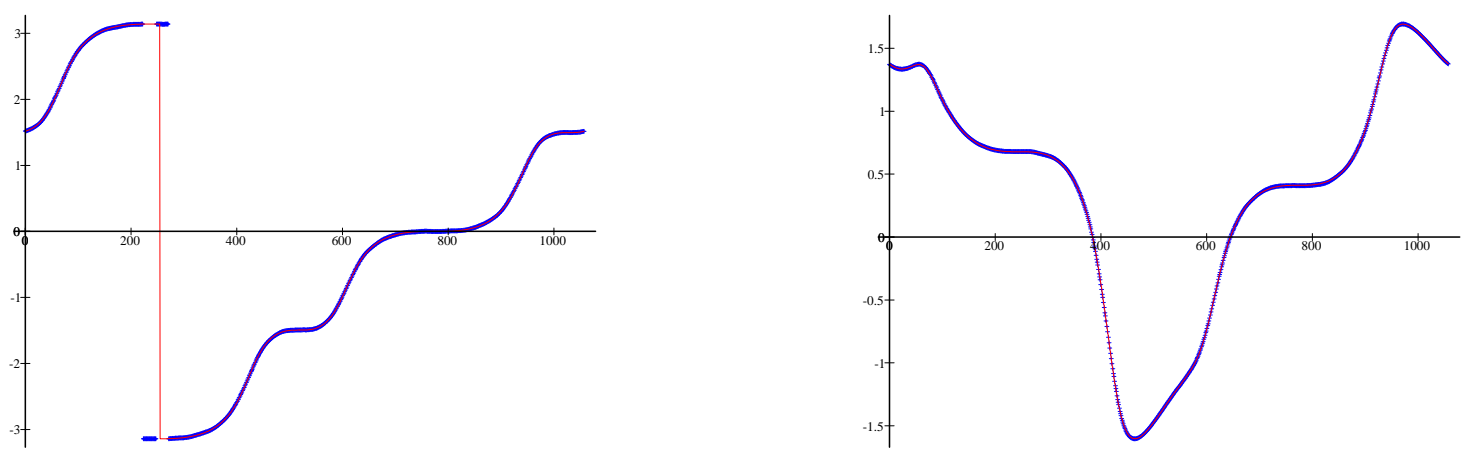

Figure 6: Estimated angle (left) and $\beta$ (right) along the curve. The X-axis is curvilinear abscissa. The crosses represent measures whereas plain curves represent theoretical values of the angle.

reduce the noise on the measured $\beta$. This is justified by the assumption that the surface $\Sigma$ is smooth enough to compute derivatives. The function $\beta(s)$ is then continuous.

The right part of Fig. 6 shows the $\beta$ estimates along the curve at one time instant for the synthetic image sequence. The maximal error between the theoric curve and the measures is 0.017152 pixels by image (the image number here is the time coordinate).

\subsection{Parameters Normalisation of System Resolution}

At this point, we have for each point of the curve $\left(c_{\tau}\right)$ all the spatio-temporal parameters we need. For each of these, we compute their normalized values. Then we build the polynomial system made from the plane equation expressed at each of these points. This system has 8 unknowns and as many equations as there are points on the edge. In order to take into account the ambiguity on $(\mathbf{V}, \mathbf{N})$ that was described in Theorem 3 we will arbitrarily normalize the first component of $\mathbf{N}$ to 1 (this choice is of course a bad choice as soon as this 


\begin{tabular}{|c|c|c|c|c|}
\hline $\begin{array}{c}\text { Number of images } \\
\text { considered }\end{array}$ & $\begin{array}{l}\mid \Omega-\Omega_{\text {theor }} \| \\
\text { in } \% \text { image }\end{array}$ & $\begin{array}{l}\Omega, \Omega_{\text {theor }} \\
\text { in }^{\circ}\end{array}$ & $\begin{array}{l}\mathbf{V}, \mathbf{V}_{\text {theor }} \\
\text { in }\end{array}$ & $\begin{array}{l}\mathbf{N}, \mathbf{N}_{\text {theor }} \\
\text { in }\end{array}$ \\
\hline 5 & $1.6 \times 10^{-1}$ & $4.1 \times 10^{-1}$ & 1.2 & 1.2 \\
\hline 13 & $2.4 \times 10^{-2}$ & 1.1 & $1.0 \times 10^{-1}$ & $6.0 \times 10^{-2}$ \\
\hline 20 & $1.4 \times 10^{-2}$ & $6.4 \times 10^{-1}$ & $6.4 \times 10^{-1}$ & $6.2 \times 10^{-2}$ \\
\hline 29 & $1.1 \times 10^{-2}$ & $5.4 \times 10^{-1}$ & $5.1 \times 10^{-1}$ & $5.6 \times 10^{-2}$ \\
\hline
\end{tabular}

Table 1: Errors in norm and angle between the estimated results and the theoretical ones as a function of the number of images used to compute the spatio-temporal parameters.

component approaches 0 ). The system we obtain has then only 7 unknowns and is solved using the method described earlier in this section.

For the synthetic sequence, using the theoretical values of the parameters we have proved that there are only two solutions to the motion problem. Namely the true solution and its companion solution as described in Theorem 4 . In what follows we will always consider errors between the true solution and the corresponding solution.

In Table 1, we have tabulated the accuracy of the computed solution for all the components of $(\boldsymbol{\Omega}, \mathbf{V}, \mathbf{N})$ as a function of the number of images of the synthetic sequence (around image 14) used to compute the spatio-temporal parameters. Note that (and this will be true for all results showed here) that for $\mathbf{V}$ and $\mathbf{N}$ only the angle between the theoretical and estimated values are shown since these values are only defined up to a scale factor. The time needed to do all the computations (including parameter estimation and resolution of the system which has 1112 equations) is about 32 seconds when 5 images are used and about 36 seconds when all 29 images are used. These times have been obtained on a Sun Sparc 2.

Figure 7 show the reconstructions for the good solution. Figure 8 shows the values of $Z$ along the curve for the good solution. Note that there are always points on the curves where the value of $Z$ computed from (4) are biased : it can be shown that these point corresponds to the points for which $\mathbf{U}_{\mathbf{t}} \cdot \mathbf{V}=0$ which can be interpreted geometrically as the points at which the tangent to 


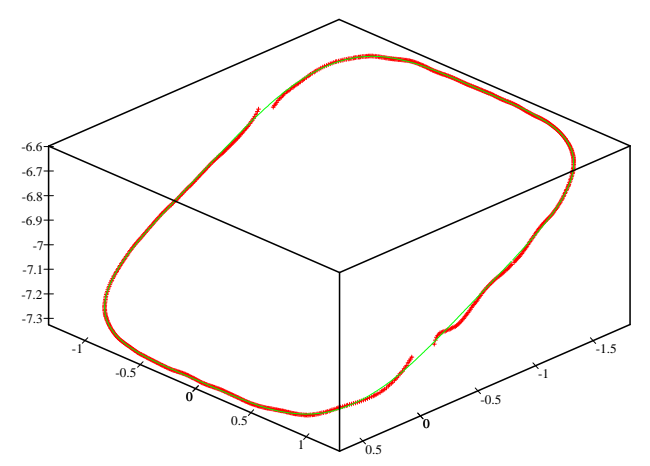

Figure 7: 3D reconstructions for the good solution. The plain curve is the reconstruction based on (5) whereas the crosses represent the reconstruction based on (4).

the curve goes through the focus of expansion. As it can be shown from (4), there is no depth information at these points.

A nice experimental consequence resulting from the comparison of the planar and general reconstruction is that it seems possible to distinguish automatically the good solution from the bad one : the good solution is always associated to the reconstructions between which the errors is the smallest. The exact explanation of this phenomena is still unclear.

Verifying quantatively the results obtained with the real sequences is a difficult task : as described in Sect. 4 the best way to do it is to look at the angular speed. With the measured data of Fig. 9 the measured angular speed is $-1.055^{\circ} /$ image where it should be $-1^{\circ}$ image. Along this sequence, the worst relative error on the angular speed is under $15 \%$ but usually this relative error is lower than $7 \%$. Note also that we have a good robustness to wrong estimation of camera parameters : we used many different internal parameters (involving changes of 5 to 10 pixels for the optical center and changes of 2 to $5 \%$ for the scale factors) obtained by calibrating with different data and noticed a 


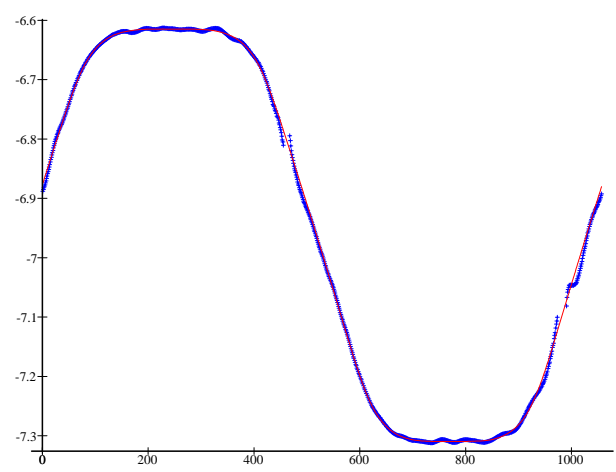

Figure 8: Estimates for $Z$ along the curve. The plain curve represent the values obtained using (5) whereas the crosses represent the values based on (4).

good stability of the computed motion (the relative error between the different parameters is at most $1 \%$ ).

Figure 9 shows the angle and beta estimates along the curve. Figure 10 shows normal flow along the curve. Figure 11 shows the reconstructions obtained from the good and the bad solutions.

\subsection{Higher Order Derivatives}

We will conclude this chapter by showing some figures giving the parameters $\kappa, \frac{\partial \beta}{\partial s}$ and $\partial_{\mathbf{n}_{\beta}} \beta$ along the curve for the synthetic image sequence. These measures seem good enough to allow a practical implementation of the stereo disambiguation described in [FP92] as well as that of the general 3D rigid curve case based on Theorem 2 .

Figures 13,14 and 15 show respectively the parameters $\kappa, \frac{\partial \beta}{\partial s}$ and $\partial_{\mathbf{n}_{\beta}} \beta$ along the observed curve. There are still some problems around curvature extrema. In fact, the origin of these problems is now well understood and will be corrected.

$\operatorname{RR} n^{\circ} 2168$ 

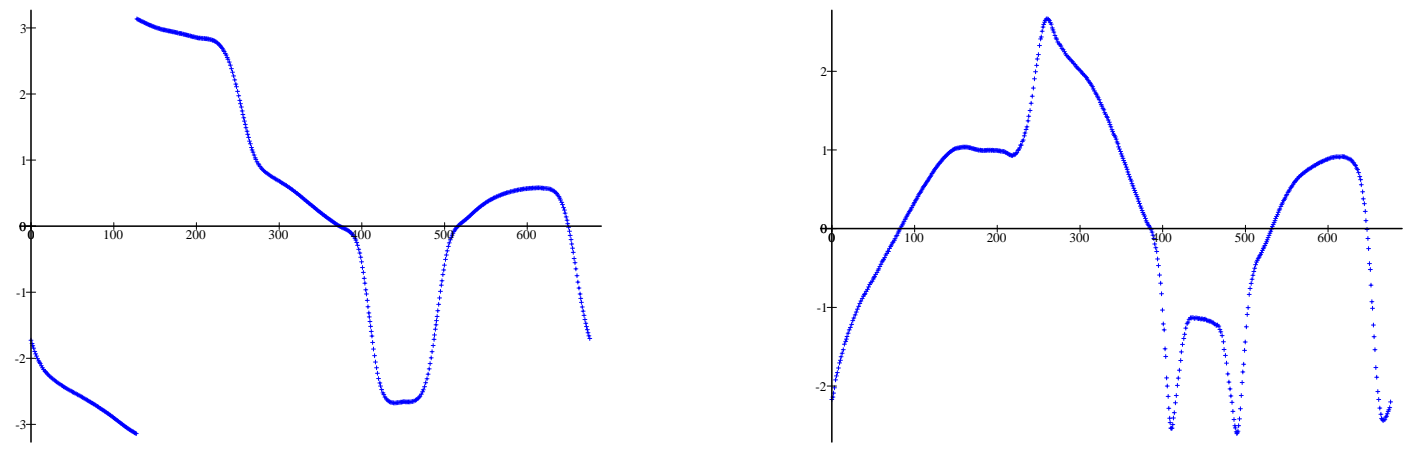

Figure 9: Estimated angle (left) and $\beta$ (right) along the curve. The X-axis is curvilinear abscissa. The crosses represent measures whereas plain curves represent theoretical values of the angle.

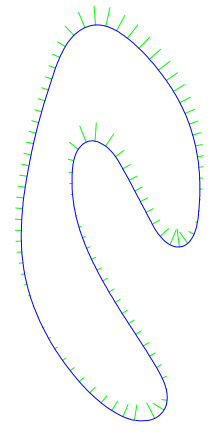

Figure 10: Normal flow along the curve. The flow has been scaled 4 times for readability. 

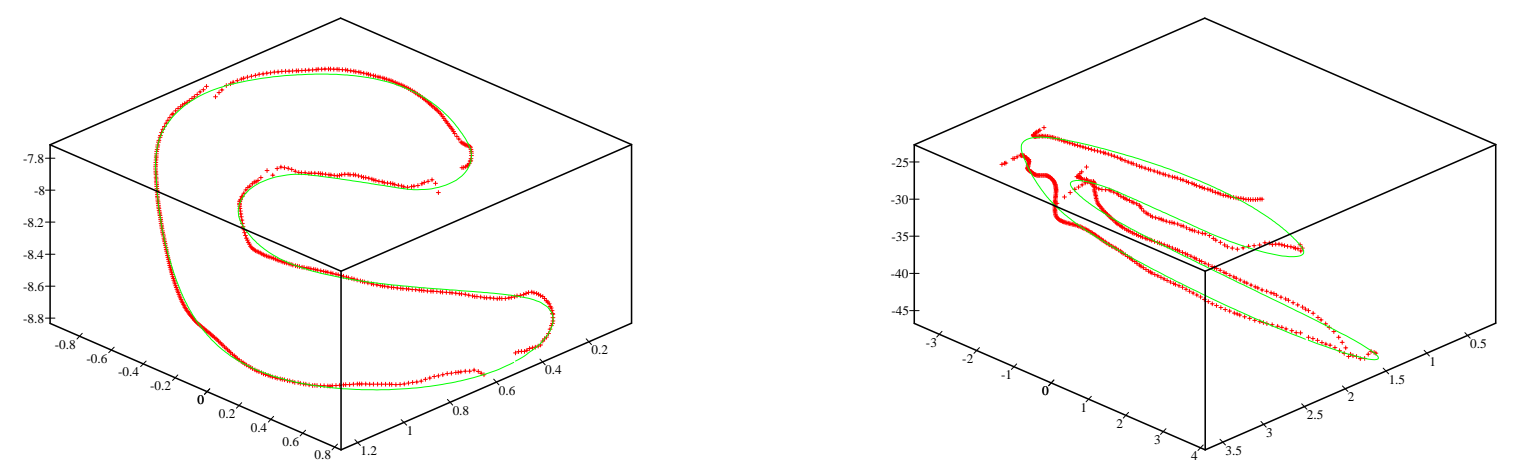

Figure 11: 3D reconstructions for the good solution (left) and the bad solution (right). The plain curve is the reconstruction based on (5) whereas the crosses represent the reconstruction based on (4).

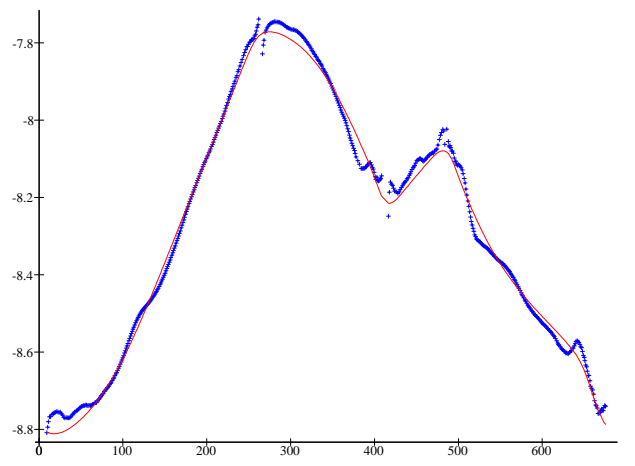

Figure 12: Estimates for $Z$ along the curve. The plain curve represent the values obtained using (5) whereas the crosses represent the values based on (4).

$\operatorname{RR~}{ }^{\circ} 2168$ 


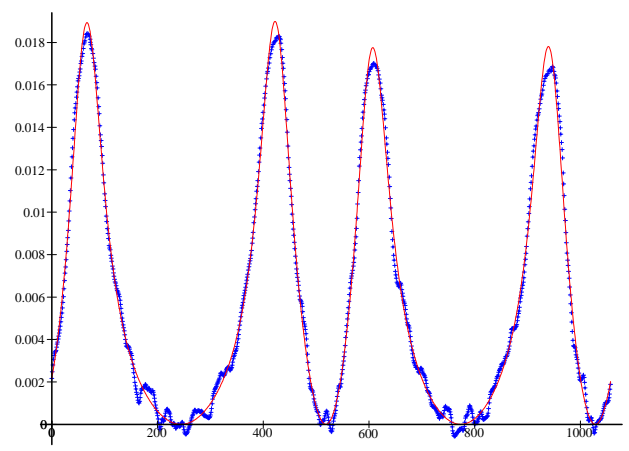

Figure 13: Measured $\kappa$ along the curve. Crosses represent measures whereas the plain curve represent the theoretical values.

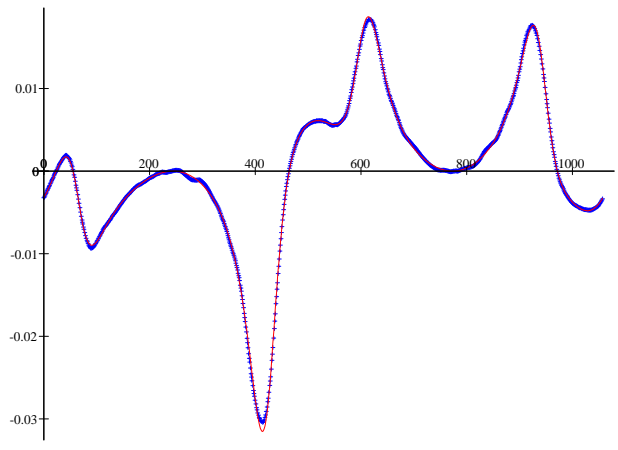

Figure 14: Estimated $\frac{\partial \beta}{\partial s}$ along the curve. Crosses represent measures whereas the plain curve represent the theoretical values. 


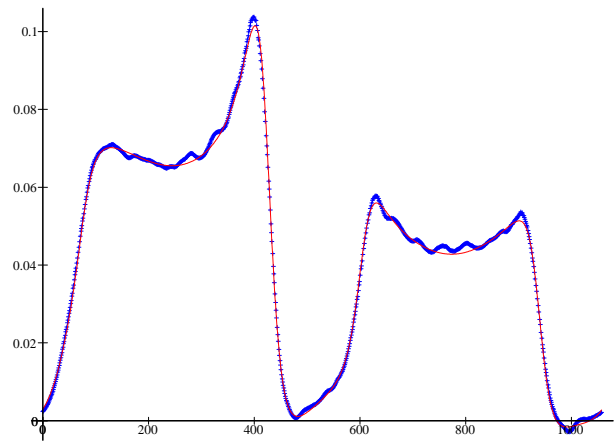

Figure 15: Estimated $\partial_{\mathbf{n}_{\beta}} \beta$ along the curve. Crosses represent measures whereas the plain curve represent the theoretical values.

$\operatorname{RR~n}{ }^{\circ} 2168$ 


\section{Conclusion and Future Work}

We have shown in this paper how one of the two equations relating the $3 \mathrm{D}$ motion of a rigid curve to the observed motion field is redundant. Moreover, we have shown that the remaining equation does not depend on the third order derivatives on the spatio-temporal surface generated by the retinal image of the 3D curve and demonstrated that the first and second order derivatives can indeed be computed quite accurately from the long image sequences. This is very promising for the implementation of that theory which is, obviously, our next goal.

From another point of view, we have completely implemented the more specific case of planar rigid $3 \mathrm{D}$ curves and shown on real and synthetic images that the theory gives quite accurate results. Moreover, it seems that the fundamental ambiguity related to the use of this special kind of curves can be overcome by looking at the errors on the $Z$ reconstruction errors between the planar and general model. This case was most fruitful since it allowed us to understand better many of the characteristics of the systems of polynomials and to develop many tools that will be useful for implementing the general case. Many improvements can still be done to improve further the quality of the results such as working with many planar curve patches or taking into account special properties of some points such as inflexion points, bitangent points or points at which the tangent to the curve goes through the focus of expansion. 


\section{References}

[AL87] N. Ayache and F. Lustman. Fast and reliable passive trinocular stereovision. In Proceedings ICCV '87, London, pages 422-427. IEEE, June 1987.

[Ber89] Fredrik Bergholm. Motion from Flow along Contours: A Note on Robustness and Ambiguous Cases. The International Journal of Computer Vision, 2(4):395-415, April 1989.

[Bou89] Patrick Bouthemy. A Maximum Likelihood Framework for Determining Moving Edges. IEEE Transactions on Pattern Analysis and Machine Intelligence, 11(5):499-511, May 1989.

[D'H86] Johan D'Hayer. Determining Motion of Image Curves from Local Pattern Changes. Computer Vision, Graphics, and Image Processing, 34:166-188, 1986.

[FDN89] Olivier D. Faugeras, Nourr-Eddine Deriche, and Nassir Navab. From optical flow of lines to 3D motion and structure. In Proceedings IEEERSJ International Workshop on Intelligent Robots and Systems '89, pages 646-649, 1989. Tsukuba, Japan.

[FLM92] Olivier D. Faugeras, Tuan Luong, and Steven Maybank. Camera self-calibration: theory and experiments. In Giulio Sandini, editor, Proceedings of the 2nd European Conference on Computer Vision, pages 321-334. Springer-Verlag, Lecture Notes in Computer Science 588, 1992.

[FLT87] Olivier D. Faugeras, Francis Lustman, and Giorgio Toscani. Motion and Structure from point and line matches. In Proceedings of the First International Conference on Computer Vision, London, pages 25-34, June 1987.

[FP92] Olivier D. Faugeras and Théo Papadopoulo. Disambiguating Stereo Matches with Spatio-Temporal Surfaces. In Joseph L. Mundy and Andrew Zimmerman, editors, Geometric Invariance in Computer Vision, chapter 16. MIT Press, 1992. 
[FT86] Olivier D. Faugeras and Giorgio Toscani. The Calibration Problem for Stereo. In Proceedings CVPR '86, Miami Beach, Florida, pages 15-20. IEEE, June 1986.

[Gon89] S. Gong. Curve Motion Constraint Equation and its Applications. In Proceedings Workshop on Visual Motion, pages 73-80, 1989. Irvine, California, USA.

[HGC92] Richard Hartley, Rajiv Gupta, and Tom Chang. Stereo from Uncalibrated Cameras. In Proceedings of CVPR92, Champaign, Illinois, pages 761-764, June 1992.

[Hil84] Ellen C. Hildreth. The Measurement of Visual Motion. MIT Press, Cambridge, Mass., 1984.

[HS81] Berthold K. P. Horn and Brian G. Schunk. Determining Optical Flow. Artificial Intelligence, 17:185-203, 1981.

[II86] M. Ito and A. Ishii. Three-View Stereo Analysis. IEEE Transactions on Pattern Analysis and Machine Intelligence, 8:524-532, 1986.

[Koe86] Jan J. Koenderink. Optic Flow. Vision Research, 26(1):161-180, 1986.

[LH84] H.C. Longuet-Higgins. The visual ambiguity of a moving plane. Proceedings of the Royal Society London, B, 223:165-175, 1984.

[LHP80] H. C. Longuet-Higgins and K. Prazdny. The interpretation of moving retinal images. Proceedings of the Royal Society of London, B 208:385-387, 1980.

[May92] S.J. Maybank. Theory of reconstruction From Image Motion. Springer-Verlag, 1992.

[MK85] V..J. Milenkovic and T. Kanade. Trinocular Vision Using Photometric and Edge Orientation Constraints. In Proceedings of DARPA Image Understanding Workshop, pages 163-175, , December 1985. 
[Nag83] H-H. Nagel. Displacement Vectors Derived from Second Order Intensity Variations in Image Sequences. Computer Vision, Graphics, and Image Processing, 21:85-117, 1983.

[PH86] M. Pietikainen and D. Harwood. Depth from three-camera stereo. In Proc. International Conference on Computer Vision and Pattern Recognition, pages 2-8. IEEE, 1986. Miami Beach, Florida.

[RF91] Luc Robert and Olivier D. Faugeras. Curve-Based Stereo: Figural Continuity And Curvature. In CVPR91, pages 57-62. IEEE, June 1991. Maui, Hawai.

[SA90] Minas E. Spetsakis and John Aloimonos. Structure from Motion Using Line Correspondences. The International Journal of Computer Vision, 4:171-183, 1990.

[Tos87] G. Toscani. Système de Calibration optique et perception du mouvement en vision artificielle. PhD thesis, Paris-Orsay, 1987.

[Tsa86] Roger Tsai. An Efficient and Accurate Camera Calibration Technique for 3D Machine Vision. In Proceedings CVPR '86, Miami Beach, Florida, pages 364-374. IEEE, June 1986.

[WKPS87] Allen M. Waxman, Behrooz Kamgar-Parsi, and Muralidhara Subbarao. Closed-Form Solutions to Image Flow Equations for 3D Structure and Motion. The International Journal of Computer Vision, 1:239-258, 1987. 
Unité de recherche INRIA Lorraine, Technôpole de Nancy-Brabois, Campus scientifique, 615 rue de Jardin Botanique, BP 101, 54600 VILLERS LES NANCY

Unité de recherche INRIA Rennes, IRISA, Campus universitaire de Beaulieu, 35042 RENNES Cedex Unité de recherche INRIA Rhône-Alpes, 46 avenue Félix Viallet, 38031 GRENOBLE Cedex 1

Unité de recherche INRIA Rocquencourt, Domaine de Voluceau, Rocquencourt, BP 105, 78153 LE CHESNAY Cedex

Unité de recherche INRIA Sophia-Antipolis, 2004 route des Lucioles, BP 93, 06902 SOPHIA-ANTIPOLIS Cedex

Éditeur

INRIA, Domaine de Voluceau, Rocquencourt, BP 105, 78153 LE CHESNAY Cedex (France)

ISSN 0249-6399 